\title{
Pathogenic PALB2 mutation in metastatic pancreatic adenocarcinoma and neuroendocrine tumour: A case report
}

\author{
DAVID CHAN ${ }^{1}$, STEPHEN CLARKE $^{1}$, ANTHONY J. GILL ${ }^{1}$, LORRAINE CHANTRILL $^{2}$, \\ JAS SAMRA ${ }^{1}$, BOB T. LI $^{3}$, TRISTAN BARNES ${ }^{1}$, KAZI NAHAR ${ }^{1}$ and NICK PAVLAKIS ${ }^{1}$ \\ ${ }^{1}$ Northern Sydney Cancer Centre, Royal North Shore Hospital, St. Leonards; ${ }^{2}$ Garvan Institute of Medical Research, \\ Darlinghurst, NSW, Australia; ${ }^{3}$ Thoracic Oncology Service, Memorial Sloan Kettering Cancer Center, New York, NY, USA
}

Received December 16, 2014; Accepted January 16, 2015

DOI: $10.3892 / \mathrm{mco} .2015 .533$

\begin{abstract}
Adenocarcinoma of the pancreas is an aggressive malignancy with poor prognosis. Pancreatic neuroendocrine tumours (PNET) comprise $\sim 3 \%$ of primary pancreatic neoplasms and they are more heterogeneous in their histological character and outcome. This is the case report of a 73-year-old female patient with synchronously diagnosed pancreatic adenocarcinoma and PNET, which is likely associated with a pathogenic partner and localizer of breast cancer 2, early onset (PALB2) mutation. The potential pathogenic significance of PALB2 and its association with various malignancies were investigated and the potential role of PALB2 in conferring sensitivity to chemotherapeutic agents, such as mitomycin $\mathrm{C}$ and cisplatin, was discussed. This case report highlights the significance of ongoing research into the molecular pathogenesis of pancreatic cancer, which may help guide the selection of optimal treatments for this disease, as well as the need for ongoing study of PALB2 as a possible predictive marker of response to DNA-damaging agents.
\end{abstract}

\section{Introduction}

Adenocarcinoma of the pancreas is an aggressive malignancy with poor prognosis. Pancreatic neuroendocrine tumours (PNET), comprising $~ 3 \%$ of primary pancreatic neoplasms (1), are more heterogeneous in their histological character and outcome. Although partner and localizer of breast cancer 2, early onset (PALB2) mutations have been associated with pancreatic adenocarcinomas (2), pancreatic adenocarcinoma coexisting with PNET is an uncommon finding. This is the presentation of a case report of synchronous diagnosis of these two malignancies in the setting of a PALB2 mutation.

Correspondence to: Dr David Chan, Northern Sydney Cancer Centre, Royal North Shore Hospital, Level 1 Acute Services Building, Reserve Road, St. Leonards, NSW 2065, Australia E-mail: dlhchan1@gmail.com

Key words: partner and localizer of breast cancer 2, early onset, pancreatic cancer, neuroendocrine tumour

\section{Case report}

A 73-year-old woman was admitted to the Royal North Shore Hospital in late 2011 in late 2011 for medical review of abdominal discomfort. Computed tomography (CT) of the abdomen and pelvis revealed masses in the pancreatic head and terminal ileum, as well as two enhancing lesions in the liver (Fig. 1). The patient underwent the Whipple's procedure, ileal resection and biopsy of the liver lesions. The histological examination demonstrated a 40-mm moderately differentiated pancreatic head adenocarcinoma (Fig. 2) arising from an intraductal papillary mucinous neoplasm, with clear margins and no nodal involvement. A low-grade neuroendocrine tumour [Ki-67<0.5\%, 1 mitosis/10 high-power fields, p4(T3N1M1); Fig. 2] was also present in the terminal ileum and aforementioned liver lesions. The patient's postoperative course was complicated by diabetes mellitus and steatorrhoea, which were well controlled with use of insulin and pancreatic enzyme supplements, respectively.

The past history of the patient notably included early left-sided breast cancer in 2007, treated by wide local excision, adjuvant radiotherapy and adjuvant tamoxifen followed by letrozole. The patient had also previously undergone laminectomy for back pain, right total knee replacement, hysterectomy and cholecystectomy. The patient had never smoked and did not drink alcohol, but her family history was notable, as her mother and sister had succumbed to pancreatic cancer and her father had succumbed to hepatocellular carcinoma.

A ${ }^{68} \mathrm{Ga}$ DOTATATE positron emission tomography (PET) scan revealed uptake by multiple liver lesions and para-aortic nodes (Fig. 3A). The patient was commenced on long-acting octreotide at a dose of $30 \mathrm{mg}$ monthly and remained well over the following year; however, a progress CT performed 15 months postoperatively revealed a lesion at the surgical margin. PET scanning demonstrated ${ }^{18} \mathrm{~F}$-fluorodeoxyglucose (FDG) but not ${ }^{68} \mathrm{Ga}$ avidity for this lesion, as well as two new hepatic lesions (Fig. 3B). The serum CA 19.9 level was 4,045 U/ml. Taken together, these findings suggested the diagnosis of metastatic pancreatic cancer.

The patient was commenced on cisplatin + gemcitabine chemotherapy in December, 2012 and tolerated treatment well, apart from dose reductions due to myelosuppression. The treatment was ceased after 5 cycles, as the patient was asymptomatic and the CA 19.9 levels had normalized. 


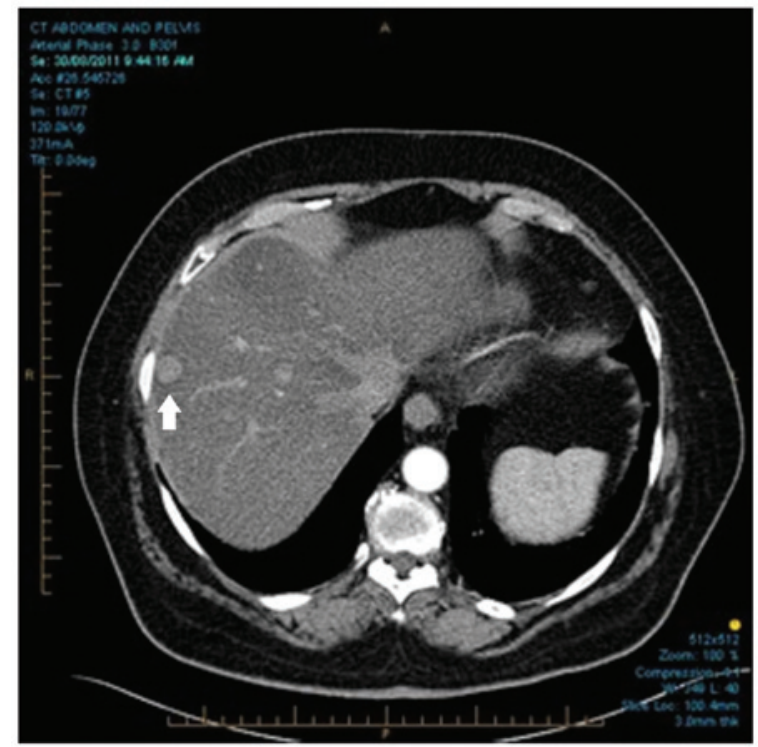

Figure 1. Computed tomography scan of the abdomen/pelvis at diagnosis (liver lesion shown with white arrow).

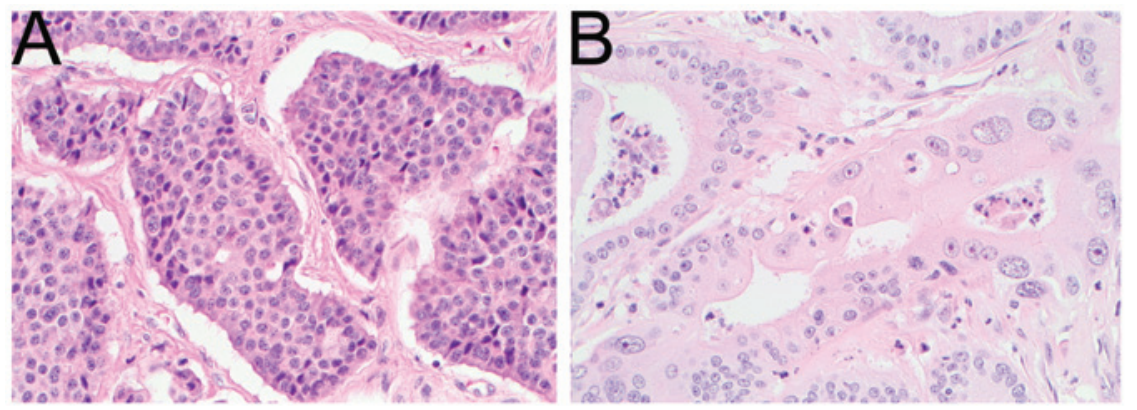

Figure 2. (A) The neuroendocrine tumour of the terminal ileum exhibited a typical morphology, including dispersed 'neuroendocrine' chromatin and a solid and trabecular growth pattern, which was very different from (B) pancreatic adenocarcinoma, which exhibited typical glandular differentiation. Hematoxylin and eosin; magnification, $\mathrm{x} 200$.
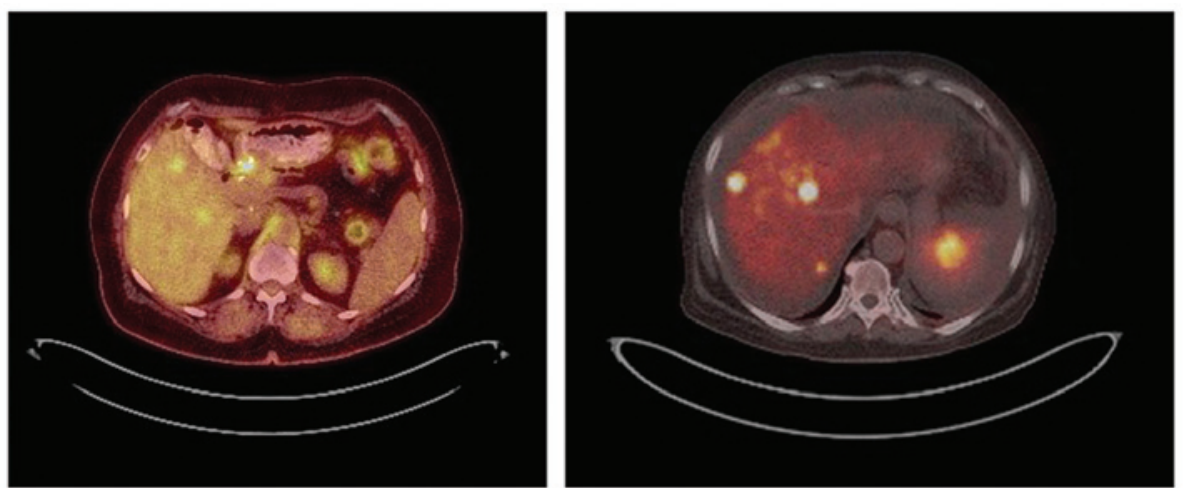

Figure 3. (A) ${ }^{68} \mathrm{Ga}$ positron emission tomography (PET) scan at diagnosis and (B) ${ }^{18} \mathrm{~F}$-fluorodeoxyglucose PET at recurrence.

New lesions were evident in the liver 2 months after cessation of treatment, with ${ }^{18} \mathrm{~F}-\mathrm{FDG}$ avidity confirmed by PET and histological confirmation of metastatic pancreatic adenocarcinoma via fine-needle biopsy. The patient was commenced on gemcitabine and capecitabine chemotherapy and subsequently received selective internal radiation therapy $\left[{ }^{90} \mathrm{Y}\right.$ microspheres - SIR-Spheres ${ }^{\circledR}$ (SIRTeX, Sydney, Australia)] to the liver.
The patient had consented to having her pancreatic tumour tissue analysed as part of the Australian Pancreatic Cancer Genome Initiative (APGI), which is a prospective study investigating broad-based sequencing of tumour and normal genomes of patients with pancreatic adenocarcinoma (3). A mutation in the PALB2 gene (PALB2 g.23, 649, 207delACAA) in the tumour was detected at that time. Further mitomycin $\mathrm{C}$ 
chemotherapy was planned; however, the patient was admitted to the hospital with Escherichia coli sepsis, delirium and gradual deterioration over the course of several weeks and succumbed to the disease in June, 2014.

The patient and her husband provided informed consent regarding the publication of the medical information.

\section{Discussion}

Pancreatic adenocarcinoma is the most common type of exocrine pancreatic malignancy and is associated with poor outcome, even following surgical resection. The 5-year survival rate from metastatic pancreatic adenocarcinoma is currently $<1 \%$ and the median survival time is $\sim 6-9$ months, even with the recent advances in chemotherapeutic regimens (4). This has prompted the search for an improved understanding of molecular pathogenesis in an attempt to identify targetable mutations underlying the development of pancreatic cancer.

Multiple germline mutations have been implicated in the pathogenesis of pancreatic cancer, such as adenomatous polyposis coli, breast cancer 1, early onset (BRCA1), BRCA2, Lynch syndrome mutations and PALB2. PALB2 mutations have been associated with malignancies of the breast (5) and pancreas, as well as non-malignant conditions, such as Fanconi anaemia (6). In a series of 1,144 familial breast cancer patients (7), 3.4\% of the patients harboured such a mutation. Although it is a recognized pathogenic mutation in familial pancreatic cancer, PALB2 mutation frequency in a series of 96 familial pancreatic cancer patients was only $3.1 \%$ (2). Mutations in the domain of PALB2 which binds BRCA2 have been shown to affect the double-stranded break repair mechanism (8), providing a rationale for chemotherapeutic agents targeting this mechanism. Xenograft models and case reports have suggested the efficacy of mitomycin $C$ or cisplatin in this setting (9).

The present case demonstrated the good clinical response of this genotype to platinum combinations. Upon reviewing other cases analysed by APGI, there are several examples of exceptional responders to platinums in the DNA damage repair pathway genotypes (10). Given the patient's family history of pancreatic cancer and personal history of breast cancer, the PALB2 mutation is almost certainly pathogenic and genetic counselling for her family has been recommended.

This case highlights the need for ongoing research into the molecular genomics of pancreatic cancer and continuous refinement of the genetic testing system to optimize treatment choices early on in diagnosis. The predictive nature of PALB2 for response to DNA-damaging chemotherapeutic agents requires further investigation.

\section{References}

1. Fesinmeyer MD, Austin MA, Li CI, et al: Differences in survival by histologic type of pancreatic cancer. Cancer Epidemiol Biomarkers Prev 14: 1766-1773, 2005.

2. Jones S, Hruban RH, Kamiyama M, et al: Exomic sequencing identifies PALB2 as a pancreatic cancer susceptibility gene. Science 324: 217, 2009.

3. Johns AL, Miller DK, Simpson SH, et al: Returning individual research results for genome sequences of pancreatic cancer. Genome Med 6: 42, 2014.

4. Von Hoff DD, Ervin T, Arena FP, et al: Increased survival in pancreatic cancer with nab-paclitaxel plus gemcitabine. N Engl J Med 369: 1691-1703, 2013.

5. Antoniou AC, Casadei S, Heikkinen T, et al: Breast-cancer risk in families with mutations in PALB2. N Engl J Med 371: 497-506, 2014.

6. Southey MC, Teo ZL and Winship I: PALB2 and breast cancer: ready for clinical translation! Appl Clin Genet 6: 43-52, 2013

7. Casadei S, Norquist BM, Walsh T, et al: Contribution of inherited mutations in the BRCA2-interacting protein PALB2 to familial breast cancer. Cancer Res 71: 2222-2229, 2011.

8. Xia B, Sheng Q, Nakanishi K, et al: Control of BRCA2 cellular and clinical functions by a nuclear partner, PALB2. Mol Cell 22: 719-729, 2006

9. Villarroel MC, Rajeshkumar NV, Garrido-Laguna I, et al: Personalizing cancer treatment in the age of global genomic analyses: PALB2 gene mutations and the response to DNA damaging agents in pancreatic cancer. Mol Cancer Ther 10: 3-8, 2011.

10. Chang DK, Grimmond SM, Evans TRJ, et al: Mining the genomes of exceptional responders. Nat Rev Cancer 14: 291-292, 2014. 